\title{
Arterias Mentolabiales Bilaterales. Reporte de un Caso
}

\author{
Bilateral Mentolabial Arteries. A Case Report
}

"Iván Claudio Suazo Galdames; "Mario Cantín López; *"Daniela Alejandra Zavando Matamala

\begin{abstract}
SUAZO, G. I. C.; CANTÍN, L. M. \& ZAVANDO, M. D. A. Arterias mentolabiales bilaterales. Reporte de un caso. Int. J. Morphol.,
\end{abstract} 26(1):43-46, 2008.

RESUMEN: En un cadáver repletado con látex coloreado rojo, se reporta la presencia bilateral de arterias ramas de la arteria facial, que se dirigen a irrigar la región mentolabial. Se discute la importancia de la descripción de las variaciones anatómicas en la irrigación de la cara y labio inferior por las implicancias en la cirugía reconstructiva de labio.

PALABRAS CLAVE: Arteria facial; Labios; Vascularización labial; Cirugía reconstructiva.

\section{INTRODUCCIÓN}

En la cirugía plástica cosmética o reconstructiva de la región mentolabial se utilizan colgajos para lo cual se requiere un detallado conocimiento de la irrigación del labio inferior.

El carcinoma de células escamosas, es una de las patologías neoplásicas más frecuentes que afectan el labio inferior en la población chilena (Riera \& Martínez, 2005). Cuando el labio inferior ha sido dañado por algún proceso tumoral, éste puede ser reconstruido utilizando colgajos miomucosos pediculados con vasos procedentes de la arteria facial. Para ello se requiere un adecuado conocimiento de la anatomía de la red arterial de las regiones labial inferior y mentoniana.

La descripción clásica indica que el labio inferior recibe irrigación a partir de una colateral anterior de la arteria facial, denominada arteria labial inferior (ALI), que se desprende de ella a la altura de la comisura oral (Testut, 1981; Rouvière \& Delmas, 1999; Figún \& Garino, 2001; Latarjet \& Ruiz-Liard, 2004), pudiendo recibir irrigación desde la arteria submentoniana (ASM), que desde la región cervical origina ramas de dirección vertical que irrigan la región mentoniana.

Koh et al. (2003) describen que la arteria facial y sus colaterales muestran importantes variaciones individuales, siendo estas arterias, las que juegan el mayor rol en la irrigación de la zona facial.
Kawai et al. (2004) describieron una serie de arterias inconstantes que se originaban de la arteria facial entre las arterias submentoniana y labial inferior, que denominaron mento-labiales horizontales (AMH), diferenciándolas de las que se originaban de la submentoniana y que llamaron mentolabiales verticales (AMV).

Estudios similares realizados por Pinar et al. (2005) denominaron arterias mentolabiales horizontales a todas aquellas que se encontraban en la región mentoniana, ellos describieron que estos vasos eran inconstantes, que se originaban de la arteria facial antes de que ella tome contacto con el músculo buccinador y que llegaban a la región, pasando profundas al músculo depresor del ángulo oral.

Edizer et al. (2003) reportaron una gran variabilidad en la arteria labial inferior, indicando que ella se encontraba unilateralmente en el $64 \%$ de los casos, y bilateralmente sólo en el $36 \%$ de los casos, encontrándose suplida por una gran cantidad de vasos de menor calibre que denominaron arterias sublabiales (ASL), a diferencia de la descripción clásica que reconoce la presencia de un círculo anastomótico perioral (Testut, Rouvière \& Delmas, Figún \& Garino, Latarjet \& Ruiz-Liard).

En este trabajo se analiza una variación anatómica en la forma en que se irriga la región mentoniana y labial inferior en un cadáver.

\footnotetext{
* Unidad de Anatomía Normal, Facultad de Ciencias de la Salud, Universidad de Talca, Chile.

** Departamento de Estomatología, Facultad de Ciencias de la Salud, Universidad de Talca, Chile.
} 


\section{REPORTE DEL CASO}

Se utilizó un cadáver adulto de sexo masculino de 55 años, de nacionalidad española, donado a la Universidad de Talca por la Universidad Cardenal Herrera de Valencia, España.

El cadáver se encontraba conservado mediante la inyección intravascular de solución fijadora conservadora en base a formaldehído y repletado su territorio arterial, mediante la canulización con un tubo plástico, con látex coloreado rojo. Se realizó la disección cuidadosa de la región facial siguiendo la arteria facial y sus colaterales; el tegumento de la región fue retirado a nivel de la tela subcutánea, desde la línea mediana hacia lateral en los lados derecho e izquierdo de la cara.

Utilizando un caliper manual $(0,5 \mathrm{~mm})$ se determinaron las características biométricas de las arterias que se encontraban irrigando las regiones mentoniana y labial inferior.

La arteria facial se originó de la carótida externa en ambos lados, luego de un trayecto cervical en el cual origina la arteria submentoniana, ésta ingresó a la región geniana pasando anterior al músculo masetero, recorrió la región geniana, profunda a los músculos risorio y cigomático mayor y superficial al buccinador, ingresando luego a la profundidad de surco nasogeniano, para constituir una variedad que Pinar et al. describen como tipo nasal de terminación, anastomosándose con las arterias del ala nasal.

Las arterias labiales inferiores se observaron en ambos lados emergiendo de la arteria facial, en el lado derecho presentó un calibre de $1,5 \mathrm{~mm}$ en su origen, a $18 \mathrm{~mm}$ del borde basilar y a $36 \mathrm{~mm}$ de la comisura oral, penetrando bajo el músculo orbicular oral a $4 \mathrm{~mm}$ de su origen (Fig. 1). En el lado izquierdo, la arteria labial inferior presentó un calibre $1,1 \mathrm{~mm}$ en su origen, levemente menor que en el lado derecho, originándose también directamente de la arteria facial, a $24 \mathrm{~mm}$ de su paso por el borde inferior de la mandíbula, a $32 \mathrm{~mm}$ de la comisura oral. La arteria fue menos sinuosa que la del lado derecho y atravesó el músculo orbicular oral a $8 \mathrm{~mm}$ de su origen (Fig 2).

En ambos lados de la región mentoniana se encontraron arterias dispuestas en sentido horizontal, interpuestas entre las labiales inferiores y la submentoniana, estas arterias llamadas mentolabiales horizontales se originaron desde la arteria facial, cruzaron inferiormente al músculo depresor del ángulo oral, al cual irrigaron, emitiendo colaterales para los músculos mentonianos, depresor del labio inferior y orbicular oral inferior.
La arteria mentolabial derecha se originó de la facial, a $7 \mathrm{~mm}$ del paso de esta arteria, por el borde inferior mandibular y a $51 \mathrm{~mm}$ de la comisura oral, su diámetro en su emergencia fue de $1 \mathrm{~mm}$ (Fig. 1).

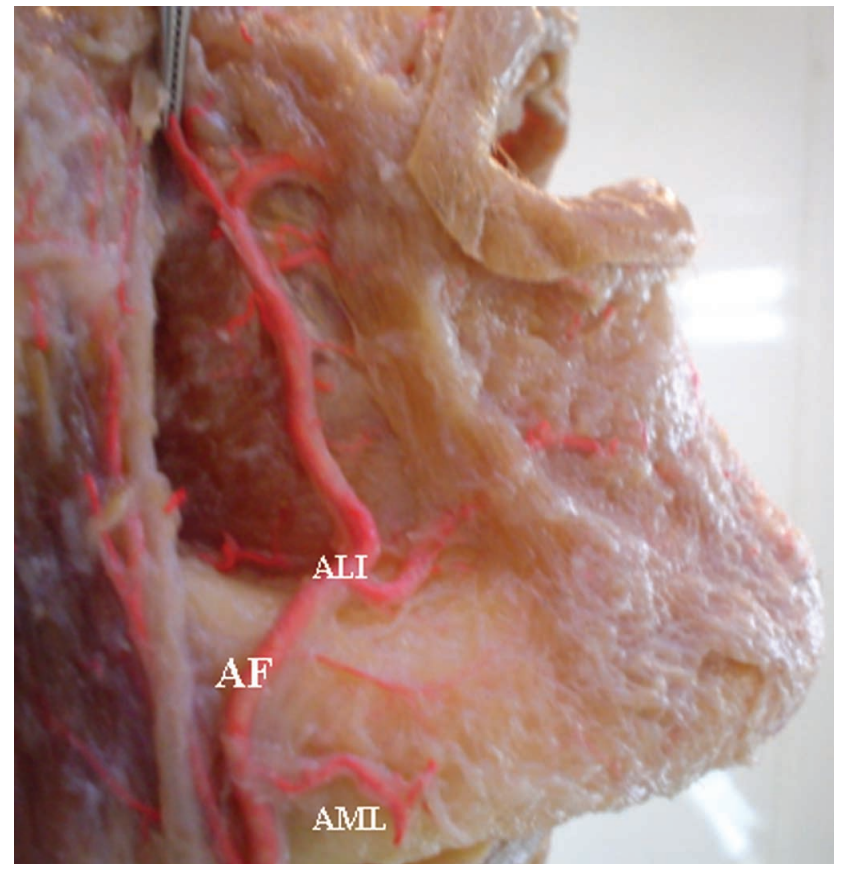

Fig. 1. Disección lado derecho. AF: arteria facial; ALI: arteria labial inferior; AML: arteria mentolabial.

La arteria mentolabial izquierda se originó de la arteria facial a $9 \mathrm{~mm}$ del paso de esta arteria, por el borde inferior mandibular y a $51 \mathrm{~mm}$ de la comisura oral, su diámetro en su origen fue de $1 \mathrm{~mm}$ (Fig 2).

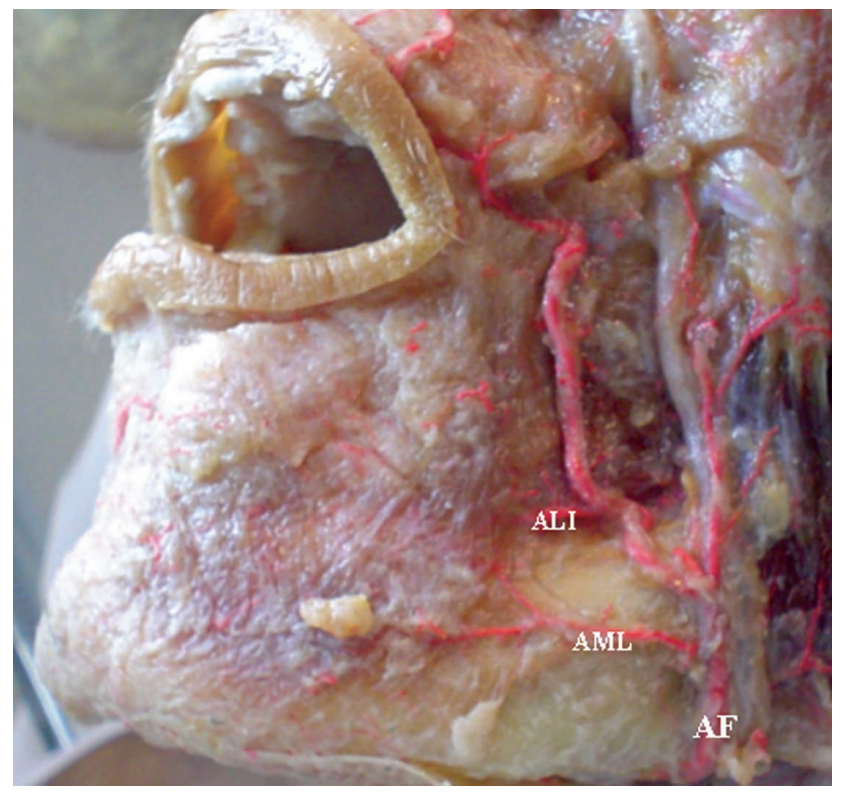

Fig 2. Disección lado izquierdo. AF: arteria facial; AML: arteria mentolabial; ALI: arteria labial inferior. 


\section{DISCUSIÓN}

En el presente reporte se analiza la presencia bilateral de las arterias mentolabiales horizontales, esta variación anatómica resulta relevante no sólo para los morfólogos sino también, para los clínicos y cirujanos que realizan procedimientos en las regiones labial y mentoniana (Park et al., 1994; Crouzet et al., 1998; Pribaz et al., 2000; Schulte et al., 2001; Gardetto et al., 2002; Cuesta et al., 2005; Gonzalez et al., 2005).

La arteria labial inferior estaba presente en ambos lados, autores como Edizer et al., indican que la forma bilateral de las arterias labiales inferiores sólo se encuentra en el $36 \%$ de los casos; el mayor calibre se encontró en el lado derecho $(+0,5 \mathrm{~mm})$, si bien en ambos casos se originaron desde la arteria facial, este origen se encontró alejado de la comisura oral (36 mm a la derecha y $32 \mathrm{~mm}$ a la izquierda), lo que no coincide con autores clásicos que señalan que las arteria labiales se desprenden de la arteria facial a la altura de la comisura oral (Tillaux, 1903; Paturet, 1951; Testut, Gardner et al., 1988; Rouvière \& Delmas, Figún \& Garino, Latarjet \& Ruiz-Liard).
La arteria mentolabial, descrita en la literatura como inconstante, se encontró de manera bilateral, con un diámetro importante ( $1 \mathrm{~mm}$ en ambos lados), lo que indica que contribuye de gran manera a la irrigación de la región mentoniana, especialmente a los músculos depresor del ángulo oral, depresor del labio, mentoniano y orbicular oral inferior.

En conclusión, la disección y ubicación de la arteria facial y de sus colaterales que irrigan la región mentolabial son importantes para los procedimientos quirúrgicos de la región mentolabial, especialmente en la cirugía reconstructiva de labio, para lo cual se han diseñado colgajos que requieren una detallada descripción de los elementos vasculares que forman sus pedículos. El conocimiento de los diámetros de estos vasos y de las distancias desde puntos de reparo estables permite un adecuado diseño de los colgajos.

La presencia de variaciones en la irrigación labial y mentoniana, como la que se describe en este caso, justifica la exploración imagenológica mediante ultrasonido o TAC Helicoidal, como paso previo a la cirugía labial reconstructiva (Wilson \& Crocker, 1985; Nagler et al., 1997; Zhao et al., 2002; Klein et al., 2003; Renshaw et al., 2007).

SUAZO, G. I. C.; CANTÍN, L. M. \& ZAVANDO, M. D. A. Bilateral mentolabial arteries. A case report. Int. J. Morphol., 26(1):43-46, 2008.

SUMMARY: In one cadaver with colored latex red injected, the presence of the facial artery branches that go to irrigate the mentolabial region is reported. One discusses to the importance of the description of the anatomical variations in the irrigation of the face and lower lip by the influence in the reconstructive surgery of lip.

KEY WORDS: Facial artery; Lips; Lip vascularisation; Reconstructive surgery.

\section{REFERENCIAS BIBLIOGRÁFICAS}

Crouzet, C.; Fournier, H.; Papon, X.; Hentati, N.; Cronier, P. \& Mercier, P. Anatomy of the arterial vascularization of the lips. Surg. Radiol. Anat., 20(4):273-8, 1998.

Cuesta, G. M.; Pujol, R. R. \& Navarro, C. C. Reconstrucción de defectos palatinos con el colgajo de músculo buccinador. Rev. Esp. Cirug. Oral y Maxilofac., 27(4):206-15, 2005.

Edizer, M.; Magden, O.; Tayfur, V.; Kiray, A.; Ergur, I. \& Atabey, A. Arterial anatomy of the lower lip: a cadaveric study. Plast. Reconstr. Surg., 111(7):2176-81, 2003.

Figún, M. \& Garino, R. Anatomía Odontológica Funcional y Aplicada. $2^{\mathrm{a}}$ ed. Buenos Aires, El Ateneo, 2001.
Gardetto, A.; Moriggl, B.; Maurer, H.; Erdinger, K. \& Papp, C. Anatomical basis for a new island axial pattern flap in the perioral region. Surg. Radiol. Anat., 24(3-4):14754, 2002.

Gardner, E.; Gray, D. J. \& O'Rahilly, R. Anatomia. 4ª ed. Rio de Janeiro, Guanabara Koogan, 1988.

Gonzalez, G. R.; Naval, G. L. \& Rodriguez, C. F. J. Colgajo miomucoso de buccinador en la reconstrucción de defectos del área maxilofacial. Rev. Esp. Cirug. Oral y Maxilofac., 27(4):197-205, 2005.

Kawai, K.; Imanishi, N.; Nakajima, H.; Aiso, S.; Kakibuchi, M. \& Hosokawa, K. Arterial anatomy of the lower lip. 
Scand. J. Plast. Reconstr. Surg. Hand Surg., 38(3):1359, 2004.

Klein, M. B.; Karanas, Y. L.; Chow, L. C.; Rubin, G. D. \& Chang, J. Early experience with computed tomographic angiography in microsurgical reconstruction. Plast. Reconstr. Surg., 112(2):498-503, 2003.

Koh, K. S.; Kim, H. J.; Oh, C. S. \& Chung, I. H. Branching patterns and symmetry of the course of the facial artery in Koreans. Int. J. Oral Maxillofac. Surg., 32(4):414-8, 2003.

Latarjet, M. \& Ruiz-Liard, A. Atlas de Anatomía. $2^{\mathrm{a}}$ ed. Buenos Aires, Panamericana, 2004.

Nagler, R. M.; Braun, J.; Daitzman, M. \& Laufer, D. Spiral CT angiography: an alternative vascular evaluation technique for head and neck microvascular reconstruction. Plast. Reconstr. Surg., 100(7):1697-702, 1997.

Park, C.; Lineaweaver, W. C. \& Buncke, H. J. New perioral arterial flaps: Anatomic study and clinical application. Plast. Reconstr. Surg., 94(2):268, 1994.

Paturet, G. Lé Traité de Anatomie Humaine. Tome I. Paris, Masson, 1951.

Pinar, Y. A.; Bilge, O. \& Govsa, F. Anatomic Study of the Blood Supply of Perioral Region. Clin. Anat., 18(5):3309, 2005.

Pribaz, J. J.; Meara, J. G. \& Wright, S. Lip and vermilion reconstruction with the facial artery musculomucosal flap. Plast. Reconstr. Surg., 105(3):864-72, 2000.

Renshaw, A.; Whitwell, K. A.; Berger, L. \& Butler, P. E. The Use of Color Doppler Ultrasound in the Assessment of Vessels for Facial Transplantation. Ann. Plast. Surg., 59(1):82-6, 2007.

Riera, P. \& Martínez, B. Morbilidad y mortalidad por cáncer oral y faríngeo en Chile. Rev. Méd. Chile, 133(5):55563, 2005.

Rouvière, H. \& Delmas, A. Anatomía Humana Descriptiva, Topográfica y Funcional. $10^{\mathrm{a}}$ ed. Barcelona, Masson, 1999. V. 3.

Schulte, D. L.; Sherris, D. A. \& Kasperbauer, J. L. The anatomical basis of the Abbé flap. Laryngoscope, 111(3):382-6, 2001.
Testut, L. Tratado de Anatomía Topográfica. $8^{\mathrm{a}}$ ed. Barcelona, Salvat, 1981.

Tillaux, P. Traité d' anatomie topographique avec applications a la chirurgie. $11^{\mathrm{a}}$ ed. Paris, Asselin et Houzeau, 1903.

Wilson, I. R. \& Crocker, E. F. An introduction to ultrasonography in oral surgery. Oral Surg. Oral Med. Oral Pathol., 59(3):236-41, 1985.

Zhao, Y. P.; Ariji, Y.; Gotoh, M.; Kurita, K.; Natsume, N.; Ma, X. C. \& Ariji, E. Color Doppler sonography of the facial artery in the anterior face. Oral Surg. Oral Med. Oral Pathol. Oral Radiol. Endod., 93(2):195-201, 2002.

Dirección para correspondencia:

Iván Claudio Suazo Galdames

Unidad de Anatomía Normal

Avenida Lircay s/n Oficina N¹04

Universidad de Talca

CHILE

Fono: 56-71-201682

Email: isuazo@utalca.cl

Recibido : 10-07-2007

Aceptado: 22-11-2007 